pathogenic variant carriers, respectively. The origin of PC is yet unknown. However, as the origin of ovarian cancer probably lies in the Fallopian tube, the Serous Tubal Intraepithelial Carcinoma (STIC) may be the origin of PC as well. In this Individual Patient Data Meta-Analysis (IPDMA), we determine the risk of PC for BRCA1/2 pathogenic variant carriers with and without STIC at RRSO.

Methodology We performed a systematic search of MEDLINE, EMBASE and Cochrane on studies providing follow-up in $B R C A$-mutation carriers after RRSO. Individual patient data was extracted and the authors of eligible studies were contacted to complete this data. Additionally, we retrospectively collected data from the Radboudumc (Nijmegen, the Netherlands), Kaiser Permanente (San Francisco, USA) and MD Anderson (Houston, USA) of BRCA1/2 pathogenic variant carriers undergoing RRSO.

Result(s)* After screening, 15 out of 2.151 studies were included. Including the retrospective case series, individual patient data was available for 3.121 women without STIC and 115 women with STIC at RRSO. The median age (range) was 46 (24-80) of women without and 52 (36-77) for women with STIC. The hazard ratio to develop PC after RRSO was 29.3 for women with STIC compared to women without STIC at RRSO $(\mathrm{P}<0.001)$ (figure 1$)$. The five-year risk to develop PC was $0.4 \%(0.2 \%-0.7 \%)$ for women without STIC and $12.6 \%(5.2 \%-19.3 \%)$ for women with STIC. The respective ten-year risks are $0.9 \%(0.3-1.4)$ and $23.5 \%$ (10.6$34.5 \%)$. Additional sensitivity analyses did not alter the results.

Conclusion* From this IPDMA we conclude that BRCA pathogenic variant carriers with a STIC at RRSO are at increased risk to develop PC during follow-up. These results are important for clinical awareness and future research. The question arises whether a STIC should be considered as precursor or early stage ovarian cancer. Larger prospective-multicenter studies are needed to investigate the additional value of staging surgery and/or chemotherapy in case of STIC.

\section{SURVIVAL IN ADVANCED-STAGE OVARIAN CARCINOMA WITH HIGH VEGF-A EXPRESSION AFTER ADDITION OF BEVACIZUMAB. RESULTS FROM THE GERMAN ICON7 PATIENT COHORT}

\footnotetext{
1,25 Kommoss ${ }^{*}, 2,3$ F Heitz, ${ }^{4}$ L Serna-Hiquita, ${ }^{2,5}$ TW Park-Simon, ${ }^{2,6,7}$ A Belau, ${ }^{2,8}$ L Hanker, ${ }^{2,9} \mathrm{KR}$ Kimmig, $2,10 \mathrm{~N}$ De Gregorio, $2,{ }^{11} \mathrm{~B}$ Schmalfeldt, ${ }^{2,12} \mathrm{~K}$ Baumann, ${ }^{2,13} \mathrm{~W}$ Schroeder, ${ }^{2,14} \mathrm{U}$ Canzler, ${ }^{2,15}$ J Sehouli, ${ }^{2,16} \mathrm{~A}$ Burges, ${ }^{2,17} \mathrm{~F}$ Hilpert, ${ }^{4} \mathrm{P}$ Martus, ${ }^{18} \mathrm{~A}$ Reuß, ${ }^{2,16} \mathrm{~S}$ Mahner, 2,3P Harter, 2,19 J Pfisterer. 'Tübingen University Hospital, Department of Women's Health, Tübingen, Germany; ${ }^{2}$ AGO Study Group, Wiesabden, Germany; ${ }^{3}$ Kliniken Essen-Mitte, Department of Gynecology and Gynecologic Oncology, Essen, Germany; ${ }^{4}$ Tuebingen University Hospital, Institut für Klinische Epidemiologie und angewandte Biometrie; ${ }^{5}$ Hannover Medical School, Hannover, Germany; ${ }^{6}$ Frauenarztpraxis Dr. Belau, Greifswald, Germany; ${ }^{7}$ University Hospital Greifswald, Greifswald, Germany; ${ }^{8}$ University Hospital Schleswig-Holstein, Campus Lübeck, Lübeck, Germany; ' University Hospital Essen, Essen, Germany; ${ }^{10}$ SLK-Kliniken Heilbronn, Klinikum am Gesundbrunnen, Gynecology and Obstetrics, Heilbronn, Germany; ${ }^{11}$ University Medical Center Hamburg-Eppendorf, Hamburg, Germany; ${ }^{12}$ Hospital Ludwigshafen, Gynecology and Obstetrics, Ludwigshafen, Germany; ${ }^{13}$ Gynaekologicum Bremen, Bremen, Germany; ${ }^{14}$ University Hospital Carl Gustav Carus, Technische Universität Dresden, Dresden, Germany; ${ }^{15}$ Charité - Universitätsmedizin Berlin, Campus Virchow, Berlin, Germany; ${ }^{16}$ University Hospital, LMU Munich, Department of Obstetrics and Gynecology, Munich, Germany; ${ }^{17}$ Krankenhaus Jerusalem Hamburg, Hamburg, Germany; ${ }^{18}$ Philipps-University Marburg, Coordinating Center for Clinical Trials, Marburg, Germany; ${ }^{19}$ Gynecologic Oncology Center, Kiel, Germany
}

Introduction/Background* It has previously been demonstrated that bevacizumab may differentially improve ovarian cancer survival. TCGA classification as well as molecular targets of anti-VEGF therapy were shown to be associated with different levels of bevacizumab efficacy. Translation into individualized treatment options or improved disease outcome was yet hindered by inconsistent results across trials and tissue types. It was the aim of this project to validate retrospective analyses of GOG-0218 derived predictive value of microvessel density (CD31) and tumor VEGF-A.

Methodology CD31 and VEGF-A immunohistochemistry was performed on whole section FFPE tissue samples from the AGO-OVAR11 (ICON7) trial. Patients were stratified into high and low biomarker-expressing subgroups using median cutoffs. The association between biomarker expression and bevacizumab therapy efficacy was evaluated using a proportional Cox regression model. Efficacy endpoints were progression free survival (PFS) and overall survival (OS).

Result(s)* Complete CD31 and VEGF-A immunhistochemical data were available from 387 patients of the German ICON7 trial cohort. Among all biomarker subgroups, only patients with high VEGF-A expression levels had a statistically significant benefit from the addition of bevacizumab to standard chemotherapy. Median PFS and OS of VEGF-A high patients was 23.1 months (95\% CI 15.9-30.2) and 64.9 months (median not reached) respectively if bevacizumab was added to standard chemotherapy but only 14.3 (95\% CI an 11.2-17) and 47.3 months (95\% CI 5.6-36.3) in the control arm. In multivariable analysis, adjusted for age, FIGO and postoperative residual tumor, the anti-angiogenic therapy showed improved PFS (HR: 0.62 [95\%CI 0.43-0.89], $\mathrm{p}=0.011$ ) and OS (HR 0.59 [95\%CI 0.39-0.91], $\mathrm{p}=0.02$ ) among VEGF-A high patients. Patients with low VEGF-A expression levels showed no statistically significant improvement of PFS (HR $0.9695 \%$ CI $0.67-1.38, \mathrm{p}=0.83$ ) or OS (HR $1.0695 \% \mathrm{CI}$ $0.69-1.62, \mathrm{p}=0.80)$ after addition of bevacizumab. CD31 immunohistochemistry was not predictive for bevacizumab treatment effects in our cohort.

Conclusion* A potential predictive value of VEGF-A expression levels was observed in advanced stage ovarian carcinoma patients from the German ICON7 patient cohort, partly confirming GOG-0218 derived findings. Our results may help to develop biomarker stratified anti-VEGF therapy and hold potential to promote personalized treatment strategies in ovarian carcinoma patients.

\section{REAL-WORLD OF OVARIAN CANCER TREATMENT OUTCOMES IN NORTHERN PORTUGAL}

J Savva-Bordalo*, M Magalhães, R Calisto, P Redondo, M Borges, A Petiz, MJ Bento, D Pereira. Porto, Medical Oncology, Porto, Portugal

\subsection{6/ijgc-2021-ESG0.472}

Introduction/Background* Epithelial ovarian cancer (EOC) is the fifth most lethal cancer in women in Portugal. Despite advances in surgical and anti-cancer systemic treatment (SACT), EOC overall prognosis remains poor. The objective of this study is to describe SACT outcomes patterns, including target therapies (bevacizumab and PARPi) using real-world data from a comprehensive cancer center, which serves Northern Portugal population. 
Methodology Retrospective observational cohort study. We reviewed medical records of diagnosed EOC patients (pts) who were eligible for SACT, between 2012 and 2018. Primary endpoint was overall survival (OS). Secondary endpoints were description of platinum sensibility patterns and lines of treatment (LOT). Descriptive analysis of main demographic, clinical and treatment variables were performed. Kaplan-Meier method was used for OS. Uni and multivariable analysis were done using Cox proportional hazard analysis

Result(s)* We identified 268 EOC pts with median age of 66 (24-94). Debulking surgery was performed in 119 pts (44.4\%). Most were stage III-IV FIGO (200, 74.6\%) and had high-grade serous morphology (103, 38.6\%). BRCA mutations (germline and/or somatic) were detected in $7.6 \%$ of 131 tested pts. A third of pts never relapsed (86, 32.1\%). Platinum-based CT was the $1^{\text {st }}$ LOT in 173 pts (64,6\%). After relapse or progression, primary platinum resistance (PPR) was present in $34(19.7 \%)$, partial platinum sensibility in 29 $(16.8 \%)$ and full platinum sensibility in 24 (13.9\%). Of the 180 pts who progressed, $41(22.8 \%)$ were submitted to $2^{\text {nd }}$ SACT and $20(11.1 \%)$ to $3^{\text {rd }}$ SACT. Median number of LOT were $2(1-8)$. Bevacizumab concomitant with CT was used in 45 pts $(16.8 \%)$ at some point. PARPi was used in $23(8.6 \%)$ pts as maintenance treatment after $\geq 2$ platinum-based CT complete or partial response. Median OS was 25.5 months [IC95\% 19.55-35.42], which was significantly worse for more advanced disease [HR 8.46 IC95\% 4.13-17.31] and PPR [HR 2.72 IC95\% 1.63-4.54].

Conclusion* Our results confirm that EOC outcomes are modest and in line with other published cohorts. Multicentric realworld studies are needed to evaluate how innovative targeted therapies, recently introduced in the daily clinical practice, will change the course of this disease.

\section{PROGNOSTIC IMPACT OF MESENTERIC LYMPH NODE METASTASIS IN PATIENTS WITH ADVANCED OVARIAN CANCER}

CF Pop*, I Veys, A El Asmar, L Polastro, J Kerger, M Chintinne, D Larsimont, G Liberale. Institut Jules Bordet, Bruxelles, Belgium

\subsection{6/ijgc-2021-ESG0.473}

Introduction/Background* Digestive tract resection is a common procedure performed in bulky advanced-stage ovarian cancer (AOC) to achieve complete cytoreductive surgery (CRS). Very few data are available on the incidence and the prognostic impact of mesenteric lymph node (MLN) involvement.

The aim of this study is to determine the incidence of MLN involvement in patients undergoing a digestive tract resection at the time of CRS for AOC and to analyze its prognostic impact.

Methodology A retrospective study including patients who underwent CRS for AOC between 2006 and 2017. Patient's clinico-pathological characteristics were collected, incidence of various LN basin involvement were analyzed focusing on MLN. Overall survival (OS) and disease free survival (DFS) were calculated with the KM method.

Result(s)* During the study period 72 of 176 (40.9\%) patients with stage III-IVa AOC had a digestive tract resection during CRS with the recto-sigmoid resection being the most frequent (52 patients). There was an involvement of the serosa in 38
$(52.8 \%)$, sub-serosa in $10(13.9 \%)$, muscularis propria in 10 (13.9\%), and mucosa in 5 cases (6.9\%), respectively. MLN were found in 37 out of $72(51.4 \%)$ patients with digestive tract resection. 25 patients $(67.5 \%)$ had MLN involvement with a median of 5 involved nodes (range, 1 to 45). There was a trend in worse 5 -year OS $(45.8 \%$ vs $63.6 \%, \mathrm{p}=0.064)$ for patients with MLN involvement.

Conclusion* Patients with AOC involving the digestive tract is associated with a high incidence of MLN metastasis. Resection of the associated lymphatic tributaries of affected bowel segment and correct pathological examination of the MLN should be performed regularly to improve staging and better evaluate their prognostic impact in case of $\mathrm{LN}$ involvement.

\section{THE ROLE OF CA-125 AND HE-4 IN EPITHELIAL OVARIAN CANCER FOLLOW-UP}

J Castella*, Á Taus, B Esteban, S Espuelas, B Fabrego, E Miralpeix Rovira, JM Sole-Sedeno, $\mathrm{G}$ Mancebo. Spain

\subsection{6/ijgc-2021-ESGO.474}

Introduction/Background* Most patients diagnosed of advanced epithelial ovarian cancer (EOC) will relapse within the first years after the treatment. It is fundamental to find a marker for recurrence monitoring. Our objective was to perform a descriptive analysis of our data to estimate the rate of recurrence of EOC and the effectiveness of Ca-125 and HE-4 to agree with the radiologic diagnostic of relapse.

Methodology All patients were diagnosed of any stage of EOC from January 2014 to June 2020. They were retrospectively selected for the assessment of HE-4 and Ca-125 levels.

All patients selected had to undergo surgical treatment with optimal cytoreduction and chemotherapy if indicated, and they had to show radiological complete response after surgery. Out of 105 patients diagnosed during this period, 79 subjects met the inclusion criteria. 7 of them were lost during the followup, so 72 patients were included in the analysis.

Result(s) $* 47$ out of 72 patients relapsed during the follow-up $(65,3 \%)$. Out of all relapses, 32 patients had available data and reached normal levels of HE-4 after the surgery. 15/32 presented positive levels of HE-4 when the relapse was diagnosed (46,9\%). Moreover, in 6/32 cases the levels of HE-4 tend to increase in the moment of the relapse, even though the value did not become positive $(18,8 \%)$.

Out of all relapses, 43 patients had available data and reached normal levels of Ca-125 after the surgery. 20 out of 43 presented positive levels of $\mathrm{Ca}-125$ when the relapse was diagnosed (46,5\%). In addition, in $15 / 43$ cases the levels of Ca-125 tend to increase in the moment of the relapse, even though the value did not become positive (34,9\%).

30 patients had available data and reached normal levels of HE-4 and Ca-125 after the surgery. 9/30 cases presented positive levels of both HE-4 and Ca-125 in the moment of relapse $(30 \%)$.

Conclusion* Most patients showed an increase of biomarkers in agreement with radiologic relapse; in half of the cases the markers level became positive, but in some patients the values tend to increase despite being in the negative range. Both markers used together seem to be useful for the followup. 